\title{
Orthogonal $m$-metric spaces and an application to solve integral equations
}

\author{
Fahim Uddin ${ }^{1}$, Choonkil Park ${ }^{2 *}$, Khalil Javed ${ }^{1}$, Muhammad Arshad ${ }^{1}$ and Jung Rye Lee ${ }^{3}$
}

"Correspondence:

baak@hanyang.ac.kr

${ }^{2}$ Research Institute for Natural

Sciences, Hanyang University, Seoul,

04763, Korea

Full list of author information is

available at the end of the article

\section{Springer}

\begin{abstract}
In this article, we introduce the concept of orthogonal $m$-metric space and prove some fixed point theorems in this space. Furthermore, we obtain results that extend and improve certain comparable results in the existing literature. Eventually, our results lead us to the existence and uniqueness of solutions for Fredholm integral equations.
\end{abstract}

MSC: Primary 05C38; 15A15; secondary 05A15; $15 \mathrm{~A} 18$

Keywords: Orthogonal set; Orthogonally complete; m-metric; Orthogonal m-metric

\section{Introdction and preliminaries}

Since the French mathematician Frechet had interpreted metric space axiomatically, developments in mathematics in general, and functional analysis in particular, took new directions in the year 1906. Looking at the subject of fixed point theory and its applications in different fields, we can see its extensiveness. Those theorems which are related to the existence of fixed points and their properties are called fixed point theorems. These theorems play a crucial role in illustrating the nature and uniqueness of solutions for various mathematical models. After the advent of the well-known Banach contraction principle, metric fixed point theory has taken new dimensions, and this contraction principle gives us a genuinely suitable basis to find a fixed point for self mapping. The Banach contraction method has played a key role in promoting metric fixed point theory in the recent past. Refer to [1-7] for more information on Banach contractions and applications. The fruitfulness of this basic principle has been proven by numerous generalizations in different directions. Inspired by this natural idea, several investigators have tried various generalizations of this notation.

In 1994, Matthews [8] introduced the notion of partial metric space. Since then, this theory has fascinated many researchers in proving the existence and solutions for various mathematical models and has a wide variety of applications in different fields related to mathematics. In 2014, Asadi et al. [9] extended the concept of a partial metric space and presented some examples to show that their definitions are a real generalization of partial metric space. They called this new notion an $m$-metric space. Later on, Patel et al. [10] extended this concept for set-valued mappings. Recently, Eshaghi Gordji et al. [11] intro-

(c) The Author(s) 2021. This article is licensed under a Creative Commons Attribution 4.0 International License, which permits use sharing, adaptation, distribution and reproduction in any medium or format, as long as you give appropriate credit to the original author(s) and the source, provide a link to the Creative Commons licence, and indicate if changes were made. The images or other third party material in this article are included in the article's Creative Commons licence, unless indicated otherwise in a credit line to the material. If material is not included in the article's Creative Commons licence and your intended use is not permitted by statutory regulation or exceeds the permitted use, you will need to obtain permission directly from the copyright holder. To view a copy of this licence, visit http://creativecommons.org/licenses/by/4.0/. 
duced the notation of orthogonal sets and gave a new extension for the classical Banach fixed point theorem and more details please refer [11-13].

In this article, we have introduced the notation of orthogonal $m_{\perp}$-metric spaces. And in this context, we have improved and generalized some well-known results in the literature and developed some fixed point theorems for the proposed contraction. Inspired by the work of many scientist [14-18] regarding fixed point theorems to prove the existence and uniqueness of solutions for certain types of integral equations related to fractional type differential equations, we have also developed an illustrative example and a possible application for the system of Fredholm integral equations to claim the validity of the proposed results.

Definition 1 ([9]) A partial metric on a nonempty set $E$ is a function $\rho: E \times E \rightarrow[0, \infty)$ such that, for all $\varpi, v, \varkappa \in E$,

$(\rho 1) \varpi=v$ if and only if $\rho(\varpi, \varpi)=\rho(\varpi, v)=\rho(v, v)$;

$(\rho 2) \rho(\varpi, \varpi) \leq \rho(\varpi, v)$;

( $\rho 3) \rho(\varpi, v)=\rho(v, \varpi)$;

$(\rho 4) \rho(\varpi, v) \leq \rho(\varpi, \varkappa)+\rho(\varkappa, v)-\rho(\varkappa, \varkappa)$.

A partial metric space is a pair $(E, \rho)$ such that $E$ is a nonempty set and $\rho$ is a partial metric on $E$.

Notation 1 The following notations are useful in the sequel:

(1) $\mu_{\varpi, v}=\min \{\mu(\varpi, \varpi), \mu(v, v)\}$.

(2) $\mu_{\varpi, v}^{\prime}=\max \{\mu(\varpi, \varpi), \mu(v, v)\}$.

Definition 2 ([10]) Let $E$ be a nonempty set. A function $\mu: E \times E \rightarrow[0, \infty)$ is called an $m$-metric if the following conditions are satisfied:

( $\mu 1) \varpi=v$ if and only if $\mu(\varpi, \varpi)=\mu(\varpi, v)=\mu(v, v)$;

( $\mu 2) \quad \mu_{\varpi, v} \leq \mu(\varpi, v)$

( $\mu 3) \mu(\varpi, v)=\mu(v, \varpi)$;

( $\mu 4) \mu(\varpi, v)-\mu_{\varpi, v} \leq\left(\mu(\varpi, \varkappa)-\mu_{\varpi, \varkappa}\right)+\left(\mu(\varkappa, v)-\mu_{\varkappa, v}\right)$

for all $\varpi, v, \varkappa \in E$.

Then the pair $(E, \mu)$ is called an $m$-metric space.

Example $1([10])$

(a) Let $E=\{1,2,3\}$. Define $\mu$ on $E \times E$ as follows:

$$
\begin{aligned}
& \mu(1,1)=1, \quad \mu(2,2)=4, \quad \mu(3,3)=5, \quad \mu(1,2)=\mu(2,1)=9, \\
& \mu(1,3)=\mu(3,1)=7, \quad \mu(3,2)=\mu(2,3)=6 .
\end{aligned}
$$

It is easy to verify that $(E, \mu)$ is an $m$-metric space but it is not a partial metric space, since $\mu(1,2) \not \leq \mu(1,3)+\mu(3,2)-\mu(3,3)$.

(b) Let $E=[0, \infty)$. Then $\mu(\varpi, v)=|\varpi-v|$ on $E$ is an $m$-metric.

Definition 3 ([19]) Let $E$ be a nonempty set. Assume that a function $\mu: E \times E \rightarrow \mathbb{R}^{+}$ satisfies the following conditions:

(1) $\varpi=v$ if and only if $\mu(\varpi, \varpi)=\mu(\varpi, v)=(v, v)$; 
(2) $\mu_{\varpi, v} \leq \mu(\varpi, v)$ for all $\varpi, v \in E$;

(3) $\mu(\varpi, v)=\mu(v, \varpi)$ for all $\varpi, v \in E$;

(4) there exists a real number $s \geq 1$ such that for all $\varpi, v, \varkappa \in E$ we have

$$
\left(\mu(\varpi, v)-\mu_{\varpi, v}\right) \leq s\left[\left(\mu(\varpi, \varkappa)-\mu_{\varpi, \varkappa}\right)+\left(\mu(\varkappa, v)-\mu_{\varkappa, v}\right)\right]-\mu(\varkappa, \varkappa) .
$$

The number $s$ is called the coefficient of the $m_{b}$-metric space. The pair $(E, \mu)$ is called an $m_{b}$-metric space.

Definition 4 ([10]) Let $(E, \mu)$ be an $m$-metric space. Then:

(1) A sequence $\left\{\varpi_{\eta}\right\}$ in an $m$-metric space $(E, \mu)$ converges to a point $\varpi \in E$ if and only if

$$
\lim _{\eta \rightarrow \infty}\left(\mu\left(\varpi_{\eta}, \varpi\right)-\mu_{\varpi_{\eta}, \varpi}\right)=0 .
$$

(2) A sequence $\left\{\varpi_{\eta}\right\}$ in an $m$-metric space $(E, \mu)$ is called $m$-Cauchy sequence if

$$
\lim _{\eta, j \rightarrow \infty}\left(\mu\left(\varpi_{\eta}, \varpi_{j}\right)-\mu_{\varpi_{\eta}, \varpi_{j}}\right) \quad \text { and } \lim _{\eta, j \rightarrow \infty}\left(\mu_{\varpi_{\eta}, \varpi_{j}}^{\prime}-\mu_{\varpi_{\eta}, \varpi_{j}}\right)
$$

exist (and are finite).

(3) An $m$-metric space $(E, \mu)$ is said to be complete if every $m$-Cauchy sequence $\left\{\varpi_{\eta}\right\}$ converges to a point $\varpi \in E$, such that

$$
\lim _{\eta \rightarrow \infty}\left(\mu\left(\varpi_{\eta}, \varpi\right)-\mu_{\varpi_{\eta}, \varpi}\right)=0 \quad \text { and } \quad \lim _{\eta \rightarrow \infty}\left(\mu_{\varpi_{\eta}, \varpi}^{\prime}-\mu_{\varpi_{\eta}, \varpi}\right)=0
$$

Definition 5 ([13]) Let $E$ be a nonempty set and $\perp$ be a binary relation defined on $E \times E$. Then $(E, \perp)$ is said to be an orthogonal set (in short, $O$-set) if there exists $\varpi_{0} \in E$, such that

$$
\left(\forall v \in E, \varpi_{0} \perp v\right) \quad \text { or } \quad\left(\forall v \in E, v \perp \varpi_{0}\right) \text {. }
$$

The element $\varpi_{0}$ is called an orthogonal element. An orthogonal set may have more than one orthogonal element.

Example 2 ([13]) Let $E=[4, \infty]$. Define $\varpi \perp v$ if there exists $\varpi \leq v$. Then by putting $\varpi_{0}=4,(E, \perp)$ is an $O$-set.

Definition $6([11])$ Let $(E, \perp)$ be an $O$-set. A sequence $\left\{\varpi_{\eta}\right\}$ is called an orthogonal sequence if

$$
\left(\forall \eta \in \mathbb{N}, \varpi_{\eta} \perp \varpi_{\eta+1}\right) \quad \text { or } \quad\left(\forall \eta \in \mathbb{N}, \varpi_{\eta+1} \perp \varpi_{\eta}\right)
$$

Definition 7 ([13]) A mapping $T: E \rightarrow E$ is orthogonal continuous (or $\perp$-continuous) in $\varpi \in E$ if for each $O$-sequence $\left\{\varpi_{\eta}\right\}_{\eta \in \mathbb{N}} \in E$ such that $\varpi_{\eta} \rightarrow \varpi, T\left(\varpi_{\eta}\right) \rightarrow T(\varpi)$. Also $T$ is said to be $\perp$-continuous on $E$ if $T$ is $\perp$-continuous at each $\varpi \in E$. 
Example $3([13])$ Let $E=[0,1)$. Suppose that

$$
\varpi \perp v \Leftrightarrow\left\{\begin{array}{c}
\varpi \leq v \leq \frac{1}{6}, \\
\varpi=0,
\end{array}\right\}
$$

is an orthogonal metric space with $\mu(\varpi, v)=\frac{\varpi+v}{2}$. If we take orthogonal sequence $\varpi_{\eta}=\frac{\eta}{2}$ for all $\eta \geq 1$, then it converges to some point that does not lie in $E$ when $\eta \rightarrow 0$. So $(E, \perp, \mu)$ is not a complete metric but if we take $\varpi=\left[0, \frac{3}{4}\right)$ and take an orthogonal subsequence of $\varpi_{\eta}$, which is $\varpi_{\eta_{z}} \leq \frac{3}{4}$ for all $\eta \geq 1$ or we take a subsequence $\varpi_{\eta_{z}}=\frac{1}{\eta}$ for all $\eta \geq 1$ then it converges to $0 \in\left[0, \frac{3}{4}\right]$ when $\eta \rightarrow \infty$. So we know that the orthogonal Cauchy sequence is convergent if its subsequence is convergent. Hence the orthogonal Cauchy sequence converges and therefore $(E, \perp, \mu)$ is an orthogonally complete metric space.

Definition 8 ([13]) Let $(E, \perp, d)$ be an orthogonal metric space. Then $X$ is said to be orthogonally complete if every Cauchy $O$-sequence is convergent.

Definition 9 ([13]) Let $(E, \perp)$ be an $O$-set. A mapping $T: E \rightarrow E$ is said to be $\perp$-preserving if $T \varpi \perp T v$ whenever $\varpi \perp v$. Also $T: E \rightarrow E$ is said to be weakly $\perp$-preserving if $T \varpi \perp$ $T v$ or $T v \perp T \varpi$ whenever $\varpi \perp v$.

Example 4 ([13]) Let $E=[0,1)$, and $\sigma_{\perp}: E \times E \rightarrow[0, \infty)$ be defined by

$$
\sigma_{\perp}(\varpi, v)=|\varpi-v|^{2}+[\max \{\varpi, v\}]^{2} \quad \text { for all } \varpi, v \in E
$$

Define $\varpi \perp v$ if $\varpi v \leq \max \left\{\frac{\varpi}{4}, \frac{v}{4}\right\}$. Then $E$ is not complete but it is an orthogonally complete metric space. Let $\varpi \perp v$ and $\varpi v \leq \frac{\varpi}{4}$. If $\left\{\varpi_{z}\right\}$ is an arbitrary Cauchy $O$-sequence in $\varpi$, then there exists a subsequence $\left\{\varpi_{z_{\eta}}\right\}$ of $\left\{\varpi_{z}\right\}$ such that $\varpi_{z_{\eta}}=0$ for all $\eta$ or there exists a subsequence $\left\{\varpi_{z_{\eta}}\right\}$ of $\left\{\varpi_{z}\right\}$ such that $\varpi_{z_{\eta}} \leq \frac{1}{4}$ for all $\eta$. It follows that $\left\{\varpi_{z_{\eta}}\right\}$ converges to a $\varpi \in[0,1)$. On the other hand, we know that every Cauchy sequence with a convergent subsequence is convergent. It follows that $\left\{E_{z}\right\}$ is convergent. Let $T: E \rightarrow E$ be a mapping defined by

$$
T(\varpi)=\left\{\begin{array}{c}
\frac{\varpi}{4}, \varpi \leq \frac{1}{4}, \\
0, \varpi>\frac{1}{4} .
\end{array}\right\}
$$

Also, $\varpi \perp v$ and $\varpi v \leq \frac{\varpi}{4}$ and so $\varpi=0$ or $v \leq \frac{1}{4}$. We have the following cases:

(a) $\varpi=0$ and $v \leq \frac{1}{4}$. Then $T(\varpi)=0$ and $T(v)=\frac{v}{4}$.

(b) $\varpi=0$ and $v>\frac{1}{4}$. Then $T(\varpi)=T(v)=0$.

(c) $v \leq \frac{1}{4}$ and $\varpi \leq \frac{1}{4}$. Then $T(v)=\frac{v}{4}$ and $T(\varpi)=\frac{\varpi}{4}$.

(d) $v \leq \frac{1}{4}$ and $\varpi>\frac{1}{4}$. Then $T(v)=\frac{v}{4}$ and $T(\varpi)=0$.

Each case implies that $T(\varpi) T(v) \leq \frac{T(\varpi)}{4}$. Hence $T$ is $\perp$-preserving.

Also, one can see that $T(\varpi)-T(v) \leq \frac{1}{4}|\varpi-v|$. Hence $T$ is a $\perp$-contraction. But $T$ is not a contraction. Otherwise, for two points $\frac{1}{4}$ and $\frac{2}{3}$ and for all $0<c<1$ we have $T\left(\frac{1}{4}\right)-T\left(\frac{2}{3}\right) \leq$ c| $\left|\frac{1}{4}-\frac{2}{3}\right|$, which is a contradiction. 
Let $\left\{\varpi_{\eta}\right\}$ be an arbitrary $O$-sequence in $\varpi$ which converges to $\varpi \in E$. Since $T$ is a $\perp$ contraction, for each $\eta \in \mathbb{N}$ we have

$$
T\left(\varpi_{\eta}\right)-T(\varpi) \leq \frac{1}{4}\left|\varpi_{\eta}-\varpi\right| .
$$

So $T$ is $\perp$-continuous. But it can be easily seen that $T$ is not continuous.

\section{Main results}

We will start this section with the definition of an $m_{\perp}$-metric space, but first we introduce the following notations, which would be helpful during the proof.

Notation 2 (1) $\sigma_{\perp \varpi, v}=\min \left\{\sigma_{\perp}(\varpi, \varpi), \sigma_{\perp}(v, v)\right\}$.

(2) $\sigma_{\perp \varpi, v}^{\prime}=\max \left\{\sigma_{\perp}(\varpi, \varpi), \sigma_{\perp}(v, v)\right\}$.

Definition 10 Let $E$ be a nonempty set. A function $\sigma_{\perp}: E \times E \rightarrow \mathbb{R}^{+}$is called an orthogonal $m_{\perp}$-metric on the orthogonal set $(E, \perp)$ if the following conditions are satisfied:

$\left(\sigma_{\perp} 1\right) \quad \varpi=v$ if and only if $\sigma_{\perp}(\varpi, \varpi)=\sigma_{\perp}(\varpi, v)=\sigma_{\perp}(v, v)$;

$\left(\sigma_{\perp} 2\right) \sigma_{\perp \varpi, v} \leq \sigma_{\perp}(\varpi, v)$ for all $\varpi, v \in E$;

$\left(\sigma_{\perp} 3\right) \quad \sigma_{\perp}(\varpi, v)=\sigma_{\perp}(v, \varpi)$ for all $\varpi, v \in E$;

$(\sigma \perp 4)$ there exists a real number $s \geq 1$ such that for all $\varpi, v, \varkappa \in E$ with $\varpi \perp \varkappa \perp v$ we have

$$
\left(\sigma_{\perp}(\varpi, v)-\sigma_{\perp \varpi, v}\right) \leq s\left[\left(\sigma_{\perp}(\varpi, \varkappa)-\sigma_{\perp \varpi, \varkappa}\right)+\left(\sigma_{\perp}(\varkappa, v)-\sigma_{\perp \varkappa, v}\right)\right]-\sigma_{\perp}(\varkappa, \varkappa) .
$$

The pair $\left(E, \sigma_{\perp}\right)$ is called an orthogonal $m_{\perp}$-metric space.

Definition 11 Let $\left(E, \sigma_{\perp}\right)$ be an orthogonal $m_{\perp}$-metric space with the coefficient $s \geq 1$.

Then:

(1) An orthogonal sequence $\left\{\varpi_{\eta}\right\}$ in an $m_{\perp}$-metric space $\left(E, \sigma_{\perp}\right)$ converges to a point $\varpi \in E$ if and only if

$$
\lim _{\eta \rightarrow \infty}\left(\sigma_{\perp}\left(\varpi_{\eta}, \varpi\right)-\sigma_{\perp \varpi_{\eta}, \varpi}\right)=0
$$

(2) An orthogonal sequence $\left\{\varpi_{\eta}\right\}$ in $E$ is said to be $m_{\perp}$-Cauchy sequence if and only if

$$
\lim _{\eta, j \rightarrow \infty}\left(\sigma_{\perp}\left(\varpi_{\eta}, \varpi_{j}\right)-\sigma_{\perp \varpi_{\eta}, \varpi_{j}}\right) \quad \text { and } \lim _{\eta, j \rightarrow \infty}\left(\sigma_{\perp \varpi_{\eta}, \varpi_{j}}^{\prime}-\sigma_{\perp \varpi_{\eta}, \varpi_{j}}\right)
$$

exist and are finite.

(3) An orthogonal $m_{\perp}$-metric space $E$ is said to be orthogonally complete if every $m_{\perp}$-Cauchy sequence $\left\{\varpi_{\eta}\right\}$ converges to a point $\varpi \in E$, such that

$$
\left(\lim _{\eta \rightarrow \infty}\left(\sigma_{\perp}\left(\varpi_{\eta}, \varpi\right)-\sigma_{\perp \varpi_{\eta}, \varpi}\right)=0 \quad \text { and } \quad \lim _{\eta \rightarrow \infty}\left(\sigma_{\perp \varpi_{\eta}, \varpi}^{\prime}-\sigma_{\perp \varpi_{\eta}, \varpi}\right)=0\right) .
$$

Definition 12 Let $\left(E, \sigma_{\perp}\right)$ be an orthogonal $m_{\perp}$-metric space. Then $T: E \rightarrow E$ is said to be orthogonally continuous (or $\perp$-continuous at $\varpi \in E$ if for each $O$-sequence $\left\{\varpi_{\eta}\right\}$ in $E$ with $\sigma_{\perp}\left(\varpi_{\eta}, \varpi\right) \rightarrow 0$, we have $\sigma_{\perp}\left(T \varpi_{\eta}, T \varpi\right) \rightarrow 0$. Also, $T$ is said to be $\perp$-continuous on $E$ if $T$ is $\perp$-continuous at each $\varpi \in E$. 
Definition 13 Let $\left(E, \sigma_{\perp}\right)$ be an orthogonal $m_{\perp}$-metric space with coefficient $s \geq 1$. Then $E$ is said to be orthogonally complete (or $O$-complete) if every Cauchy $O$-sequence is convergent in $E$.

Theorem 1 Let $\left(E, \sigma_{\perp}\right)$ be an $O$-complete $m_{\perp}$-metric space with coefficient $s \geq 1$ and $T$ : $E \rightarrow E$ be $\perp$-preserving, and $\perp$-continuous and satisfy the following condition:

$$
\sigma_{\perp}(T \varpi, T v) \leq z \sigma_{\perp}(\varpi, v) \quad \text { for all } \varpi, v \in E \text { with } \varpi \perp v
$$

where $z \in[0,1)$. Then $T$ has a unique fixed point $\varpi \in E$ and $\sigma_{\perp}(\varpi, \varpi)=0$.

Proof By the definition of orthogonality, there exists $\varpi_{0} \in E$ such that $\left(\forall v \in E, \varpi_{0} \perp v\right)$ or $\left(\forall v \in E, v \perp \varpi_{0}\right)$. It follows that $\varpi_{0} \perp T \varpi_{0}$ or $T \varpi_{0} \perp \varpi_{0}$. Let $\varpi_{1}=T \varpi_{0}, \varpi_{2}=T \varpi_{1}$, $\varpi_{3}=T \varpi_{2}, \varpi_{4}=T \varpi_{3}, \ldots, \varpi_{\eta+1}=T \varpi_{\eta}$, for all $\eta \in \mathbb{N}$. Since $T$ is $\perp$-preserving, $\left\{\varpi_{\eta}\right\}$ is $O$ sequence. Then by $(2.1)$, we get

$$
\sigma_{\perp}\left(\varpi_{\eta}, \varpi_{\eta+1}\right)=\sigma_{\perp}\left(T \varpi_{\eta-1}, T \varpi_{\eta}\right) \leq z^{\eta} \sigma_{\perp}\left(\varpi_{0}, \varpi_{1}\right)
$$

for all $\eta \in \mathbb{N}$. For any two positive integers $j>\eta$, we have

$$
\begin{aligned}
\sigma_{\perp}\left(\varpi_{\eta}, \varpi_{j}\right) \leq \sigma_{\perp}\left(T \varpi_{\eta-1}, T \varpi_{j-1}\right) \\
\leq z \sigma_{\perp}\left(\varpi_{\eta-1}, \varpi_{j-1}\right) \\
\leq z \sigma_{\perp}\left(z \sigma_{\perp}\left(\varpi_{\eta-2}, \varpi_{j-2}\right)\right) \\
\leq z^{2} \sigma_{\perp}\left(\varpi_{\eta-2}, \varpi_{j-2}\right) \\
\\
\vdots \\
\leq \\
\leq z^{\eta} \sigma_{\perp}\left(\varpi_{0}, \varpi_{j-\eta}\right) .
\end{aligned}
$$

Hence

$$
\begin{aligned}
\sigma_{\perp} & \left(\varpi_{\eta}, \varpi_{j}\right)-\sigma_{\perp \varpi_{\eta}, \varpi_{j}} \\
& \leq z^{\eta}\left(s \sigma_{\perp}\left(\varpi_{0}, \varpi_{1}\right)+s \sigma_{\perp}\left(\varpi_{1}, \varpi_{j-\eta}\right)\right) \\
& \leq z^{\eta}\left(s \sigma_{\perp}\left(\varpi_{0}, \varpi_{1}\right)+s^{2} \sigma_{\perp}\left(\varpi_{1}, \varpi_{2}\right)+s^{2} \sigma_{\perp}\left(\varpi_{2}, \varpi_{j-\eta}\right)\right) \\
& \leq z^{\eta}\left(s \sigma_{\perp}\left(\varpi_{0}, \varpi_{1}\right)+s^{2} \sigma_{\perp}\left(\varpi_{1}, \varpi_{2}\right)+\cdots+s^{j-\eta} \sigma_{\perp}\left(\varpi_{j-\eta-1}, \varpi_{j-\eta}\right)\right) \\
& \leq z^{\eta}\left(s \sigma_{\perp}\left(\varpi_{0}, \varpi_{1}\right)+z^{\eta} s^{2} z \sigma_{\perp}\left(\varpi_{0}, \varpi_{1}\right)+\cdots+z^{\eta} s^{j-\eta-1} z^{j-1} \sigma_{\perp}\left(\varpi_{0}, \varpi_{1}\right)\right) \\
& \leq s z^{\eta}\left(1+s z+(s z)^{2}+\cdots+\cdots\right) \sigma_{\perp}\left(\varpi_{0}, \varpi_{1}\right) \\
& =\frac{s z^{\eta}}{1-s z} \sigma_{\perp}\left(\varpi_{0}, \varpi_{1}\right) .
\end{aligned}
$$

Since $z \in[0,1)$ and $s>0$, it follows from the above inequality that

$$
\sigma_{\perp}\left(\varpi_{\eta}, \varpi_{j}\right)-\sigma_{\perp \varpi_{\eta}, \varpi_{j}} \rightarrow 0 \quad \text { as } \eta, j \rightarrow \infty .
$$


Similarly,

$$
\sigma_{\perp}^{\prime}\left(\varpi_{\eta}, \varpi_{j}\right)-\sigma_{\perp \varpi_{\eta}, \varpi_{j}} \rightarrow 0 \quad \text { as } \eta, j \rightarrow \infty
$$

and so $\sigma_{\perp \varpi_{\eta}, \varpi} \rightarrow 0$ as $\eta \rightarrow \infty$. Hence we have $\sigma_{\perp}\left(\varpi_{\eta}, \varpi\right) \rightarrow 0$ as $\eta \rightarrow \infty . \sigma_{\perp}(\varpi, \varpi)=0=$ $\sigma_{\perp \varpi_{\eta}, \omega}$. Thus $\left\{\varpi_{\eta}\right\}$ is an $m_{\perp}$-Cauchy sequence in $E$. Since $E$ is $O$-complete, there exists $\varpi \in E$ such that

$$
\sigma_{\perp}\left(\varpi_{\eta}, \varpi\right)-\sigma_{\perp \varpi_{\eta}, \varpi} \rightarrow 0 \quad \text { as } \eta \rightarrow \infty
$$

Now we show that $E$ is a fixed point of $T$ in $E$.

$$
\begin{aligned}
\sigma_{\perp}(\varpi, T \varpi) & \leq \lim _{\eta \rightarrow \infty} \sup \left(\sigma_{\perp}\left(\varpi, \varpi_{\eta}\right)\right)+\lim _{\eta \rightarrow \infty} \sup \left(\sigma_{\perp}\left(\varpi_{\eta}, T \varpi\right)\right) \\
& =\lim _{\eta \rightarrow \infty} \sup \sigma_{\perp}\left(\varpi_{\eta}, T \varpi\right) \\
& =\lim _{\eta \rightarrow \infty} \sup \sigma_{\perp}\left(T \varpi_{\eta-1}, T \varpi\right) \\
& \leq \lim _{\eta \rightarrow \infty} \sup z \sigma_{\perp}\left(\varpi_{\eta-1}, \varpi\right)
\end{aligned}
$$

and by $\left(\sigma_{\perp} 4\right)$ we have

$$
\begin{aligned}
& \sigma_{\perp}(\varpi, T \varpi) \\
& \quad \leq \lim _{\eta \rightarrow \infty} \sup z\left[\left(\sigma_{\perp}\left(\varpi_{\eta-1}, T \varpi\right)-\sigma_{\perp \varpi_{\eta-1}, T \varpi}\right)+\left(\sigma_{\perp}(T \varpi, \varpi)-\sigma_{\perp T \varpi, \varpi}\right)\right] \\
& \quad-\sigma_{\perp}(T \varpi, T \varpi) \\
& \leq z \sigma_{\perp}(T \varpi, \varpi) .
\end{aligned}
$$

So

$$
\begin{aligned}
& \sigma_{\perp}(\varpi, T \varpi)=0, \\
& 0 \leq \sigma_{\perp}(T \varpi, T \varpi) \leq z \sigma_{\perp}(\varpi, \varpi)=0 .
\end{aligned}
$$

Thus

$$
\sigma_{\perp}(T \varpi, T \varpi)=\sigma_{\perp}(\varpi, T \varpi)=\sigma_{\perp}(\varpi, \varpi) .
$$

By $\left(\sigma_{\perp} 1\right)$, we get $T \varpi=\varpi$. Hence $\varpi$ is a fixed point of $T$.

To prove the uniqueness property of fixed point, let $v \in E$ be another fixed point of $T$. Then we have $T^{\eta} \varpi=\varpi, T^{\eta} v=v$ for all $\eta \in \mathbb{N}$. By the choice of $\varpi_{0}$ in the first part of the proof, we obtain

$$
\left[\varpi_{0} \perp \varpi \text { and } \varpi_{0} \perp v\right] \quad \text { or } \quad\left[\varpi \perp \varpi_{0} \text { and } v \perp \varpi_{0}\right]
$$

Since $T$ is $\perp$-preserving, we have

$$
\left[T^{\eta} \varpi_{0} \perp T^{\eta} \varpi \text { and } T^{\eta} \varpi_{0} \perp T^{\eta} v\right]
$$


or

$$
\left[T^{\eta} \varpi \perp T^{\eta} \varpi_{0} \text { and } T^{\eta} v \perp T^{\eta} \varpi_{0}\right]
$$

for all $\eta \in \mathbb{N}$. Thus

$$
\sigma_{\perp}(\varpi, v)=\sigma_{\perp}\left(T^{\eta} \varpi, T^{\eta} v\right) \leq z \sigma_{\perp}(\varpi, v)<\sigma_{\perp}(\varpi, v)
$$

which implies that

$$
\sigma_{\perp}(\varpi, v)=0
$$

and so

$$
\varpi=v \text {. }
$$

Finally, we show that if $\varpi$ is a fixed point then $\sigma_{\perp}(\varpi, v)=0$. Assume that $\varpi$ is a fixed point of $T$. Then

$$
\sigma_{\perp}(\varpi, \varpi)=\sigma_{\perp}\left(T^{\eta} \varpi, T^{\eta} v\right) \leq z \sigma_{\perp}(\varpi, \varpi)<\sigma_{\perp}(\varpi, \varpi)
$$

that is, $\sigma_{\perp}(\varpi, \varpi)=0$.

Example 5 Let $E=[0,10]$, and $\sigma_{\perp}: E \times E \rightarrow \mathbb{R}^{+}$be given by

$$
\sigma_{\perp}(\varpi, v)=|\varpi-v|^{2}
$$

for all $\varpi, v \in E$. Define the binary relation $\perp$ on $E$ by $\varpi \perp v$ if $\varpi v \leq 4 \varpi$. Then $\left(E, \sigma_{\perp}\right)$ is an $O$-complete $m_{\perp}$-metric space. Define the mapping $T: E \rightarrow E$ by

$$
T(\varpi)= \begin{cases}\frac{\varpi}{4}, & 0 \leq \varpi \leq 4, \\ 0, & 4<\varpi \leq 10 .\end{cases}
$$

Let $\varpi \perp v$. Without loss of generality, we may assume that $\varpi v \leq 4 \varpi$. Then the following cases are satisfied:

(a) If $\varpi=0$ and $0 \leq v \leq 4$, then $T(\varpi)=0$ and $T(v)=\frac{v}{4}$.

(b) If $\varpi=0$ and $4<v \leq 10$, then $T(\varpi)=T(v)=0$.

(c) If $0 \leq v \leq 1$ and $0 \leq \varpi \leq 4$, then $T(v)=\frac{v}{4}$ and $T(\varpi)=\frac{\varpi}{4}$.

(d) If $0 \leq v \leq 1$ and $4<\varpi \leq 10$, then $T(v)=\frac{v}{4}$ and $T(\varpi)=0$.

These cases imply that $T(\varpi) T(v) \leq 4 T(\varpi)$. Hence $T$ is $\perp$-preserving.

Also, one can see that $|T(\varpi)-T(v)|^{2} \leq \frac{1}{16}|\varpi-v|^{2}$ for all $\varpi, v \in E$ with $\varpi \perp v$. Hence $T$ is a $\perp$-contraction with $z=\frac{1}{16}$. But $T$ is not a contraction. Otherwise, for two points $\frac{19}{5}$ and $\frac{28}{5}$ and for all $0<c<0.25$ we have $\left|T\left(\frac{19}{5}\right)-T\left(\frac{28}{5}\right)\right|^{2} \leq c\left|\frac{19}{5}-\frac{28}{5}\right|^{2}$.

Let $\left\{\varpi_{\eta}\right\}$ be an arbitrary $O$-sequence in $E$ such that $\left\{\varpi_{\eta}\right\}$ converges to $\varpi \in E$. Since $T$ is a $\perp$-contraction, for each $\eta \in \mathbb{N}$ we have

$$
\left|T\left(\varpi_{\eta}\right)-T(\varpi)\right|^{2} \leq \frac{1}{16}\left|\varpi_{\eta}-\varpi\right|^{2}
$$


As $\eta$ goes to infinity, $T$ is $\perp$-continuous, but it can be easily seen that $T$ is not continuous. Therefore all the conditions of Theorem 1 are satisfied. Hence we can conclude that $T$ has a unique fixed point in $E$, that is, a point 0 .

Theorem 2 Let $\left(E, \sigma_{\perp}\right)$ be an O-complete $m_{\perp}$-metric space with coefficient $s \geq 1$ and $T$ : $E \rightarrow E$ be $\perp$-preserving, $\perp$-contraction and $\perp$-continuous and satisfy

$$
\sigma_{\perp}(T \varpi, T v) \leq z\left[\sigma_{\perp}(\varpi, T \varpi)+\sigma_{\perp}(v, T v)\right] \text { for all } \varpi, v \in E \text { with } \varpi \perp v,
$$

where $z \in\left[0, \frac{1}{s}\right)$. Then $T$ has a unique fixed point $\varpi \in E$ and $\sigma_{\perp}(\varpi, \varpi)=0$.

Proof By the definition of orthogonality, there exists $\varpi_{0} \in E$ such that $\left(\forall v \in E, \varpi_{0} \perp v\right)$ or $\left(\forall v \in E, v \perp \varpi_{0}\right)$. It follows that $\varpi_{0} \perp T \varpi_{0}$ or $T \varpi_{0} \perp \varpi_{0}$. Let $\varpi_{1}=T \varpi_{0}, \varpi_{2}=T \varpi_{1}$, $\varpi_{3}=T \varpi_{2}, \varpi_{4}=T \varpi_{3}, \ldots, \varpi_{\eta+1}=T \varpi_{\eta}$, for all $\eta \in \mathbb{N}$. Since $T$ is $\perp$-preserving, $\left\{\varpi_{\eta}\right\}$ is an $\mathrm{O}$-sequence. Then by (2.2), we get

$$
\begin{aligned}
\sigma_{\perp}\left(\varpi_{\eta}, \varpi_{\eta+1}\right) & =\sigma_{\perp}\left(T \varpi_{\eta-1}, T \varpi_{\eta}\right) \leq z\left[\sigma_{\perp}\left(\varpi_{\eta-1}, T \varpi_{\eta-1}\right)+\sigma_{\perp}\left(\varpi_{\eta}, T \varpi_{\eta}\right)\right] \\
& =z\left[\sigma_{\perp}\left(\varpi_{\eta-1}, \varpi_{\eta}\right)+\sigma_{\perp}\left(\varpi_{\eta}, \varpi_{\eta+1}\right)\right]
\end{aligned}
$$

for all $\eta \in \mathbb{N} . \sigma_{\perp}\left(\varpi_{\eta}, \varpi_{\eta+1}\right) \leq z \sigma_{\perp}\left(\varpi_{\eta-1}, \varpi_{\eta}\right)+z \sigma_{\perp}\left(\varpi_{\eta}, \varpi_{\eta+1}\right)$, which implies $\sigma_{\perp}\left(\varpi_{\eta}\right.$, $\left.\varpi_{\eta+1}\right) \leq \eta \sigma_{\perp}\left(\varpi_{\eta-1}, \varpi_{\eta}\right)$, where $k=\frac{z}{1-z}<1$ as $z \in\left[0, \frac{1}{s}\right)$. By repeating this process, we get

$$
\sigma_{\perp}\left(\varpi_{\eta}, \varpi_{\eta+1}\right) \leq k^{\eta} \sigma_{\perp}\left(\varpi_{0}, \varpi_{1}\right)
$$

So $\lim _{\eta \rightarrow \infty} \sigma_{\perp}\left(\varpi_{\eta}, \varpi_{\eta+1}\right)=0$. By $(2.3)$, for any two natural numbers $j>\eta$,

$$
\begin{aligned}
\sigma_{\perp}\left(\varpi_{\eta}, \varpi_{j}\right) & \leq \sigma_{\perp}\left(T \varpi_{\eta-1}, T \varpi_{j-1}\right) \\
& \leq z\left[\sigma_{\perp}\left(\varpi_{\eta-1}, T \varpi_{\eta-1}\right)+\sigma_{\perp}\left(\varpi_{j-1}, T \varpi_{j-1}\right)\right] \\
& =z\left[\sigma_{\perp}\left(\varpi_{\eta-1}, \varpi_{\eta}\right)+\sigma_{\perp}\left(\varpi_{j-1}, \varpi_{j}\right)\right]
\end{aligned}
$$

and so $\lim _{\eta \rightarrow \infty} \sigma_{\perp}\left(\varpi_{\eta}, \varpi_{j}\right)=0$. Thus, for every $\xi>0$, we can find a natural number $\eta_{0}$ such that $\sigma_{\perp}\left(\varpi_{\eta}, \varpi_{\eta+1}\right)<\frac{\xi}{2}$ and $\sigma_{\perp}\left(\varpi_{j}, \varpi_{j+1}\right)<\frac{\xi}{2}$ for all $\eta, j>\eta_{0}$. Therefore, it follows that

$$
\sigma_{\perp}\left(\varpi_{\eta}, \varpi_{j}\right) \leq z\left[\sigma_{\perp}\left(\varpi_{\eta-1}, \varpi_{\eta}\right)+\sigma_{\perp}\left(\varpi_{j-1}, \varpi_{j}\right)\right]<z\left[\frac{\xi}{2}+\frac{\xi}{2}\right]<\frac{\xi}{2}+\frac{\xi}{2}=\xi \quad \text { for all } \eta, j>\eta_{0},
$$

and $\sigma_{\perp}\left(\varpi_{\eta}, \varpi_{j}\right) \rightarrow 0$ as $\eta, j \rightarrow \infty$. Hence

$$
\sigma_{\perp}\left(\varpi_{\eta}, \varpi_{j}\right)-\sigma_{\perp \varpi_{\eta}, \varpi_{j}}<\xi \quad \text { for all } \eta, j>\eta_{0} .
$$

Now for all natural numbers $\eta$, $j$, we have

$$
\begin{aligned}
\sigma_{\perp}\left(\varpi_{\eta}, \varpi_{\eta}\right) & =\sigma_{\perp}\left(T \varpi_{\eta-1}, T \varpi_{\eta-1}\right) \\
& \leq z\left[\sigma_{\perp}\left(\varpi_{\eta-1}, T \varpi_{\eta-1}\right)+\sigma_{\perp}\left(\varpi_{\eta-1}, T \varpi_{\eta-1}\right)\right] \\
& =z\left[\sigma_{\perp}\left(\varpi_{\eta-1}, \varpi_{\eta}\right)+\sigma_{\perp}\left(\varpi_{\eta-1}, \varpi_{\eta}\right)\right]
\end{aligned}
$$




$$
\leq 2 z \sigma_{\perp}\left(\varpi_{\eta-1}, \varpi_{\eta}\right) \rightarrow 0 \quad \text { as } \eta \rightarrow \infty
$$

and so $\sigma_{\perp}\left(\varpi_{\eta}, \varpi_{\eta}\right) \rightarrow 0$, which implies that

$$
\sigma\left(\varpi_{\eta}, \varpi_{j}\right)-\sigma_{\perp \varpi_{\eta}, \varpi_{j}}<\xi \quad \text { for all } \eta, j>\eta_{0}
$$

Thus $\left\{\varpi_{\eta}\right\}$ is an orthogonal $m_{\perp}$-Cauchy sequence in $E$ and

$$
\lim _{\eta, j \rightarrow \infty} \sigma_{\perp}\left(\varpi_{\eta}, \varpi_{j}\right)-\sigma_{\perp \varpi_{\eta}, \varpi_{j}}=0
$$

Thus $\left\{\varpi_{\eta}\right\}$ is an $m_{\perp}$-Cauchy sequence in $E$. Since $E$ is $O$-complete, there exists $\varpi \in E$ such that

$$
\begin{aligned}
& \sigma_{\perp}\left(\varpi_{\eta}, \varpi\right)-\sigma_{\perp \varpi_{\eta}, \varpi} \rightarrow 0 \text { as } \eta \rightarrow \infty \\
& \sigma_{\perp}(\varpi, \varpi)=0=\sigma_{\perp \varpi, T \varpi} .
\end{aligned}
$$

Now we show that $\varpi$ is a fixed point of $T$ in $E$ :

$$
\begin{aligned}
\sigma_{\perp}(\varpi, T \varpi) & \leq \lim _{\eta \rightarrow \infty} \sup \left(\sigma_{\perp}\left(\varpi, \varpi_{\eta}\right)\right)+\lim _{\eta \rightarrow \infty} \sup \left(\sigma_{\perp}\left(\varpi_{\eta}, T \varpi\right)\right) \\
& =\lim _{\eta \rightarrow \infty} \sup \sigma_{\perp}\left(\varpi_{\eta}, T \varpi\right) \\
& \leq \lim _{\eta \rightarrow \infty} \sup \left[z\left(\sigma_{\perp}\left(\varpi_{\eta-1}, T \varpi_{\eta-1}\right)+\sigma_{\perp}(\varpi, T \varpi)\right)\right] \\
& \leq \lim _{\eta \rightarrow \infty} \sup z \sigma_{\perp}\left(\varpi_{\eta-1}, \varpi_{\eta}\right)+\lim _{\eta \rightarrow \infty} \sup z \sigma_{\perp}(\varpi, T \varpi) \\
& \leq z \sigma_{\perp}(\varpi, T \varpi) .
\end{aligned}
$$

So

$$
\begin{aligned}
& \sigma_{\perp}(\varpi, T \varpi)=0, \\
& 0 \leq \sigma_{\perp}(T \varpi, T \varpi) \leq 2 z \sigma_{\perp}(\varpi, \varpi)=0 .
\end{aligned}
$$

Thus we have

$$
\sigma_{\perp}(T \varpi, T \varpi)=\sigma_{\perp}(\varpi, T \varpi)=\sigma_{\perp}(\varpi, \varpi)
$$

By $\left(\sigma_{\perp} 1\right)$, we get $T \varpi=\varpi$. Hence $\varpi$ is a fixed point of $T$.

To prove the uniqueness property of fixed point, let $v \in E$ be another fixed point of $T$. Then we have $T^{\eta} \varpi=\varpi, T^{\eta} v=v$ for all $\eta \in \mathbb{N}$. By the choice of $\varpi_{0}$ in the first part of the proof, we obtain

$$
\left[\varpi_{0} \perp \varpi \text { and } \varpi_{0} \perp v\right] \quad \text { or } \quad\left[\varpi \perp \varpi_{0} \text { and } v \perp \varpi_{0}\right]
$$

Since $T$ is $\perp$-preserving, we have

$$
\left[T^{\eta} \varpi_{0} \perp T^{\eta} \varpi \text { and } T^{\eta} \varpi_{0} \perp T^{\eta} v\right]
$$


or

$$
\left[T^{\eta} \varpi \perp T^{\eta} \varpi_{0} \text { and } T^{\eta} v \perp T^{\eta} \varpi_{0}\right]
$$

for all $\eta \in \mathbb{N}$. Thus

$$
\begin{aligned}
\sigma_{\perp}(\varpi, v) & =\sigma_{\perp}\left(T^{\eta} \varpi, T^{\eta} v\right) \\
& \leq z\left[\sigma_{\perp}(\varpi, T \varpi)+\sigma_{\perp}(v, T v)\right] \\
& =z\left[\sigma_{\perp}(\varpi, \varpi)+\sigma_{\perp}(v, v)\right]=0
\end{aligned}
$$

which implies that

$$
\sigma_{\perp}(\varpi, v)=0
$$

and so

$$
\varpi=v \text {. }
$$

Finally, we show that if $\varpi$ is a fixed point then $\sigma_{\perp}(\varpi, v)=0$. Assume that $\varpi$ is a fixed point of $T$. Then

$$
\begin{aligned}
\sigma_{\perp}(\varpi, \varpi) & =\sigma_{\perp}\left(T^{\eta} \varpi, T^{\eta} \varpi\right) \\
& \leq z\left[\sigma_{\perp}(\varpi, T \varpi)+\sigma_{\perp}(\varpi, T \varpi)\right] \\
& =2 z \sigma_{\perp}(\varpi, T \varpi) \\
& =2 z \sigma_{\perp}(\varpi, \varpi),
\end{aligned}
$$

that is, $\sigma_{\perp}(\varpi, \varpi)=0$.

Theorem 3 Let $\left(E, \sigma_{\perp}\right)$ be any $O$-complete $m_{\perp}$-metric space with coefficient $s \geq 1$ and $T$ : $E \rightarrow E$ be $\perp$-preserving, and $\perp$-continuous mapping satisfying the following conditions:

$$
\sigma_{\perp}(T \varpi, T v) \leq z \max \left\{\sigma_{\perp}(\varpi, v), \sigma_{\perp}(\varpi, T \varpi), \sigma_{\perp}(v, T v\}\right.
$$

for all $\varpi, v \in E$, where $z \in\left[0, \frac{1}{s}\right)$. Then $T$ has a unique fixed point $\varpi^{*} \in E$ and $\sigma_{\perp}\left(\varpi^{*}, \varpi^{*}\right)=0$.

Proof By the definition of orthogonality, there exists $\varpi_{0} \in E$ such that $\left(\forall v \in E, \varpi_{0} \perp v\right)$ or $\left(\forall v \in E, v \perp \varpi_{0}\right)$. It follows that $\varpi_{0} \perp T \varpi_{0}$ or $T \varpi_{0} \perp \varpi_{0}$. Let $\varpi_{1}=T \varpi_{0}, \varpi_{2}=$ $T \varpi_{1}, \ldots, \varpi_{\eta+1}=T \varpi_{\eta}$ for all $\eta \in \mathbb{N}$. Since $T$ is $\perp$-preserving, $\left\{E_{\eta}\right\}$ is an $O$-sequence. Then by (2.4), we have

$$
\begin{aligned}
\sigma_{\perp}\left(\varpi_{\eta+1}, \varpi_{\eta}\right) & =\sigma_{\perp}\left(T \varpi_{\eta}, T \varpi_{\eta-1}\right) \\
& \leq z \max \left\{\sigma_{\perp}\left(\varpi_{\eta}, \varpi_{\eta-1}\right), \sigma_{\perp}\left(\varpi_{\eta}, T \varpi_{\eta}\right), \sigma_{\perp}\left(\varpi_{\eta-1}, T \varpi_{\eta-1}\right)\right\} \\
& =z \max \left\{\sigma_{\perp}\left(\varpi_{\eta}, \varpi_{\eta-1}\right), \sigma_{\perp}\left(\varpi_{\eta}, \varpi_{\eta+1}\right), \sigma_{\perp}\left(\varpi_{\eta-1}, \varpi_{\eta}\right)\right\}
\end{aligned}
$$




$$
=z \max \left\{\sigma_{\perp}\left(\varpi_{\eta}, \varpi_{\eta-1}\right), \sigma_{\perp}\left(\varpi_{\eta}, \varpi_{\eta+1}\right)\right\} .
$$

If $\max \left\{\sigma_{\perp}\left(\varpi_{\eta}, \varpi_{\eta-1}\right), \sigma_{\perp}\left(\varpi_{\eta}, \varpi_{\eta+1}\right)\right\}=\sigma_{\perp}\left(\varpi_{\eta+1}, \varpi_{\eta}\right)$, then, from the above inequality, we obtain $\sigma_{\perp}\left(\varpi_{\eta+1}, \varpi_{\eta}\right) \leq z \sigma_{\perp}\left(\varpi_{\eta+1}, \varpi_{\eta}\right)<\sigma_{\perp}\left(\varpi_{\eta+1}, \varpi_{\eta}\right)$, which is a contradiction. Therefore, we must have $\max \left\{\sigma_{\perp}\left(\varpi_{\eta}, \varpi_{\eta-1}\right), \sigma_{\perp}\left(\varpi_{\eta}, \varpi_{\eta+1}\right)\right\}=\sigma_{\perp}\left(\varpi_{\eta}, \varpi_{\eta-1}\right)$ and again from the above inequality, we have

$$
\sigma_{\perp}\left(\varpi_{\eta+1}, \varpi_{\eta}\right) \leq z \sigma_{\perp}\left(\varpi_{\eta}, \varpi_{\eta-1}\right) .
$$

Repeating this process, we obtain

$$
\sigma_{\perp}\left(\varpi_{\eta+1}, \varpi_{\eta}\right) \leq z^{\eta} \sigma_{\perp}\left(\varpi_{1}, \varpi_{0}\right)
$$

for all $\eta \geq 0$. Thus

$$
\begin{aligned}
\sigma_{\perp} & \left(\varpi_{\eta}, \varpi_{j}\right)-\sigma_{\perp \varpi_{\eta}, \varpi_{j}} \\
& \leq z^{\eta}\left(s \sigma_{\perp}\left(\varpi_{0}, \varpi_{1}\right)+s \sigma_{\perp}\left(\varpi_{1}, \varpi_{j-\eta}\right)\right) \\
& \leq z^{\eta}\left(s \sigma_{\perp}\left(\varpi_{0}, \varpi_{1}\right)+s^{2} \sigma_{\perp}\left(\varpi_{1}, \varpi_{2}\right)+s^{2} \sigma_{\perp}\left(\varpi_{2}, \varpi_{j-\eta}\right)\right) \\
& \leq z^{\eta}\left(s \sigma_{\perp}\left(\varpi_{0}, \varpi_{1}\right)+s^{2} \sigma_{\perp}\left(\varpi_{1}, \varpi_{2}\right)+\cdots+s^{j-\eta} \sigma_{\perp}\left(\varpi_{j-\eta-1}, \varpi_{j-\eta}\right)\right) \\
& \leq z^{\eta}\left(s \sigma_{\perp}\left(\varpi_{0}, \varpi_{1}\right)+z^{\eta} s^{2} z \sigma_{\perp}\left(\varpi_{0}, \varpi_{1}\right)+\cdots+z^{\eta} s^{j-\eta-1} z^{j-1} \sigma_{\perp}\left(\varpi_{0}, \varpi_{1}\right)\right) \\
& \leq s z^{\eta}\left(1+s z+(s z)^{2}+\cdots+\cdots\right) \sigma_{\perp}\left(\varpi_{0}, \varpi_{1}\right) \\
& =\frac{s z^{\eta}}{1-s z} \sigma_{\perp}\left(\varpi_{0}, \varpi_{1}\right) .
\end{aligned}
$$

Since $z \in\left[0, \frac{1}{s}\right)$ and $s>0$, it follows from the above inequality that

$$
\sigma_{\perp}\left(\varpi_{\eta}, \varpi_{j}\right)-\sigma_{\perp \varpi_{\eta}, \varpi_{j}} \rightarrow 0 \quad \text { as } \eta, j \rightarrow \infty .
$$

Similarly,

$$
\sigma_{\perp}^{\prime}\left(\varpi_{\eta}, \varpi_{j}\right)-\sigma_{\perp \varpi_{\eta}, \varpi_{j}} \rightarrow 0 \quad \text { as } \eta, j \rightarrow \infty
$$

and so $\sigma_{\perp \varpi_{\eta}, \varpi} \rightarrow 0$ as $\eta \rightarrow \infty$. Hence we have $\sigma_{\perp}\left(\varpi_{\eta}, \varpi\right) \rightarrow 0$ as $\eta \rightarrow \infty . \sigma_{\perp}(\varpi, \varpi)=0=$ $\sigma_{\perp \varpi_{\eta}, \varpi}$. Thus $\left\{\varpi_{\eta}\right\}$ is an $m_{\perp}$-Cauchy sequence in $E$. Since $E$ is $O$-complete, there exists $\varpi \in E$ such that

$$
\sigma_{\perp}\left(\varpi_{\eta}, \varpi\right)-\sigma_{\perp \varpi_{\eta}, \varpi} \rightarrow 0 \quad \text { as } \eta \rightarrow \infty
$$

Now we show that $\varpi$ is a fixed point of $T$ in $E$ :

$$
\begin{aligned}
\sigma_{\perp}(\varpi, T \varpi) & \leq \lim _{\eta \rightarrow \infty} \sup \left(\sigma_{\perp}\left(\varpi, \varpi_{\eta}\right)\right)+\lim _{\eta \rightarrow \infty} \sup \left(\sigma_{\perp}\left(\varpi_{\eta}, T \varpi\right)\right) \\
& =\lim _{\eta \rightarrow \infty} \sup \sigma_{\perp}\left(\varpi_{\eta}, T \varpi\right) \\
& =\lim _{\eta \rightarrow \infty} \sup \sigma_{\perp}\left(T \varpi_{\eta-1}, T \varpi\right)
\end{aligned}
$$




$$
\begin{aligned}
& \leq \lim _{\eta \rightarrow \infty} \sup \left[z \sigma_{\perp}\left(\varpi_{\eta-1}, \varpi\right), \sigma_{\perp}\left(\varpi_{\eta-1}, T \varpi_{\eta-1}\right), \sigma_{\perp}(\varpi, T \varpi)\right] \\
& \leq \lim _{\eta \rightarrow \infty} \sup z \sigma_{\perp}\left(\varpi_{\eta-1}, \varpi\right), \lim _{\eta \rightarrow \infty} \sup z \sigma_{\perp}\left(\varpi_{\eta-1}, \varpi_{\eta}\right), \lim _{\eta \rightarrow \infty} \sup z \sigma_{\perp}(\varpi, T \varpi) \\
& \leq z \sigma_{\perp}(\varpi, T \varpi) .
\end{aligned}
$$

So

$$
\begin{aligned}
& \sigma_{\perp}(\varpi, T \varpi)=0, \\
& 0 \leq \sigma_{\perp}(T \varpi, T \varpi) \leq z \max \left\{\sigma_{\perp}(\varpi, \varpi), \sigma_{\perp}(\varpi, T \varpi), \sigma_{\perp}(\varpi, T \varpi)\right\}=\sigma_{\perp}(\varpi, \varpi)=0 .
\end{aligned}
$$

Thus

$$
\sigma_{\perp}(T \varpi, T \varpi)=\sigma_{\perp}(\varpi, T \varpi)=\sigma_{\perp}(\varpi, \varpi) .
$$

By $\left(\sigma_{\perp} 1\right)$, we get $T \varpi=\varpi$. Hence $\varpi$ is a fixed point of $T$.

To prove the uniqueness property of fixed point, let $v^{*} \in E$ be another fixed point of $T$. Then we have $T^{\eta} \varpi^{*}=\varpi^{*}, T^{\eta} v^{*}=v^{*}$ for all $\eta \in \mathbb{N}$. By the choice of $\varpi_{0}$ in the first part of the proof, we obtain

$$
\left[\varpi_{0} \perp \varpi^{*} \text { and } \varpi_{0} \perp v^{*}\right] \quad \text { or } \quad\left[\varpi^{*} \perp \varpi_{0} \text { and } v^{*} \perp \varpi_{0}\right] \text {. }
$$

Since $T$ is $\perp$-preserving, we have

$$
\left[T^{\eta} \varpi_{0} \perp T^{\eta} \varpi^{*} \text { and } T^{\eta} \varpi_{0} \perp T^{\eta} v^{*}\right]
$$

or

$$
\left[T^{\eta} \varpi^{*} \perp T^{\eta} \varpi_{0} \text { and } T^{\eta} v^{*} \perp T^{\eta} \varpi_{0}\right]
$$

for all $\eta \in \mathbb{N}$. Hence we get

$$
\begin{aligned}
\sigma_{\perp}\left(\varpi^{*}, v^{*}\right) & =\sigma_{\perp}\left(T^{\eta} \varpi^{*}, T^{\eta} v^{*}\right) \leq z \max \left\{\sigma_{\perp}\left(\varpi^{*}, v^{*}\right), \sigma_{\perp}\left(\varpi^{*}, T \varpi^{*}\right), \sigma_{\perp}\left(v^{*}, T v^{*}\right)\right\} \\
& =z \max \left\{\sigma_{\perp}\left(\varpi^{*}, v^{*}\right), \sigma_{\perp}\left(\varpi^{*}, \varpi^{*}\right), \sigma_{\perp}\left(v^{*}, v^{*}\right)\right\} \\
& =z \sigma_{\perp}\left(\varpi^{*}, v^{*}\right) \\
& <\sigma_{\perp}\left(\varpi^{*}, v^{*}\right)
\end{aligned}
$$

which is a contradiction. Therefore, we must have $\sigma_{\perp}\left(\varpi^{*}, v^{*}\right)=0$, that is, $\varpi^{*}=v^{*}$. Thus if a fixed point of $T$ exists then it is unique.

\section{An application to integral equation}

In this section, we endeavor to apply Theorem 1 to investigate the existence and uniqueness of solution of the Fredholm integral equation. Consider the space $E=C(I)$ of continuous functions defined on $I=[0,1]$ with the $m_{\perp}$-metric given by

$$
\sigma_{\perp}(\varpi(t), v(t))=\sup _{t \in[a, b]}\left(\left|\frac{\varpi(t)+v(t)}{2}\right|\right)
$$


for all $\varpi, v \in C(I)$. This space can also be equipped with an orthogonal given by $\varpi, v \in$ $C(I), \varpi \perp v \Leftrightarrow \varpi \leq v$. Now, we consider the following Fredholm type integral equation:

$$
\varpi(t)=\int_{0}^{1} G(t, s, \varpi(t)) d s \quad \text { for } t, s \in[0,1],
$$

where $G \in C([0,1], \mathbb{R})$.

Then $\left(E, \sigma_{\perp}\right)$ is an orthogonal complete $m_{\perp}$-metric space.

Now, we are going to state and prove our result.

Theorem 4 Assume that, for all $\varpi, v \in C([0,1], \mathbb{R})$,

$$
|G(t, s, \varpi(t))+G(t, s, v(t))| \leq z|\varpi(t)+v(t)|, \quad \text { for all } t, s \in[0,1]
$$

where $z \in[0,1)$. Then the integral equation (3.1) has a unique solution.

Proof Define $T: E \rightarrow E$ by

$$
T \varpi(t)=\int_{0}^{1} G(t, s, \varpi(t)) d s, \quad \text { for all } t, s \in[0,1] .
$$

Observe that existence of a fixed point of the operator $T$ is equivalent to the existence of a solution of the integral equation (3.1). Now, for all $\varpi, v \in E$, we have

$$
\begin{aligned}
\sigma_{\perp}(T \varpi, T v) & =\left|\frac{T \varpi(t)+T v(t)}{2}\right|=\left|\int_{0}^{1}\left(\frac{G(t, s, \varpi(t))+G(t, s, v(t))}{2}\right) d s\right| \\
& \leq \int_{0}^{1}\left|\left(\frac{G(t, s, \varpi(t))+G(t, s, v(t))}{2}\right)\right| d s \\
& \leq z \int_{0}^{1}\left|\frac{\varpi(t)+v(t)}{2}\right| d s \\
& \leq z \sup _{t \in[a, b]}\left(\frac{\varpi(t)+v(t)}{2}\right) \int_{0}^{1} d s \\
& \leq z \sigma_{\perp}(\varpi, v) .
\end{aligned}
$$

Thus the condition (2.1) is satisfied. Therefore, all the conditions of Theorem 1 are satisfied. Hence the operator has a unique fixed point, which means that the Fredholm integral equation (3.1) has a unique solution. This completes the proof.

\section{Conclusion and future work}

In this article, we have introduced the concept of orthogonal $m_{\perp}$-metric space and proved some fixed point theorems in this space. Furthermore, we have obtained results that extend and improve certain comparable results in the existing literature. Eventually, our results lead to the existence and uniqueness of solutions for Fredholm integral equations. As future directions of the studies on the introduced new space, new fixed point results can be investigated for the non-unique fixed points of self-mappings. More precisely, geometric properties of the set $\operatorname{Fix}(T)$ can be investigated as a future problem for a self-mapping $T$ on an orthogonal $m_{\perp}$-metric space in the case of non-unique fixed point. 


\section{Acknowledgements}

We would like to express our sincere gratitude to the anonymous referee for his/her helpful comments that will help to improve the quality of the manuscript.

Funding

Not applicable.

Availability of data and materials

Not applicable.

\section{Competing interests}

The authors declare that they have no competing interests.

\section{Authors' contributions}

The authors equally conceived of the study, participated in its design and coordination, drafted the manuscript, participated in the sequence alignment, and read and approved the final manuscript.

\section{Author details}

${ }^{1}$ Department of Mathematics, International Islamic University, Islamabad, Pakistan. ${ }^{2}$ Research Institute for Natural

Sciences, Hanyang University, Seoul, 04763, Korea. ${ }^{3}$ Department of Mathematics, Daejin University, Kyunggi, 11159, Korea.

\section{Publisher's Note}

Springer Nature remains neutral with regard to jurisdictional claims in published maps and institutional affiliations.

\section{Received: 14 October 2020 Accepted: 25 February 2021 Published online: 06 March 2021}

\section{References}

1. Banach, S.: Sur les operations dans les ensembles abstraits et leur application aux equations integrales. Fundam. Math. 3, 133-181 (1922)

2. Barakat, M.A., Aydi, H., Mukheimer, A., Soliman, A.A., Hyder, A.: On multivalued L-contractions and an application. Adv. Differ. Equ. 2020, Article ID 554 (2020)

3. Karapinar, E., Fulga, A., Aydi, H.: Study on Pata E-contractions. Adv. Differ. Equ. 2020, Article ID 539 (2020)

4. Czerwik, S.: Contraction mappings in b-metric spaces. Acta Math. Inform. Univ. Ostrav. 1, 5-11 (1993)

5. Mustafa, Z., Roshan, J.R., Parvaneh, V., Kadelburg, Z.: Common fixed point results in ordered partial $b$-metric spaces. J. Inequal. Appl. 2013, Article ID 562 (2013)

6. Shukla, S.: Partial $b$-metric spaces and fixed point theorems. Mediterr. J. Math. 11, 703-711 (2014)

7. Yamaod, O., Sintunavarat, W.: On new orthogonal contractions in b-metric spaces. Int. J. Pure Math. 5, 37-40 (2018)

8. Matthews, S.G.: Partial metric topology. Ann. N.Y. Acad. Sci. 728, 183-197 (1994)

9. Asadi, M., Karapinar, E., Salimi, P.: New extension of $p$-metric spaces with some fixed point results on $M$-metric spaces. J. Inequal. Appl. 2014, Article ID 18 (2014)

10. Patle, P.R., Patel, D.K., Aydi, H., Gopal, D., Mlaiki, N.: Nadlr and Kannan type set valued mappings in M-metric spaces and an application. Mathematics 7, Article ID 373 (2019)

11. Eshaghi Gordji, M., Ramezani, M., de La Sen, M., Cho, Y:: On orthogonal sets and Banach fixed point theorem. Fixed Point Theory 18, 569-578 (2017)

12. Baghani, H., Eshagahi Gordji, M., Ramezani, M.: Orthogonal sets: the axiom of choice and proof of a fixed point theorem. J. Fixed Point Theory Appl. 18, 465-477 (2016)

13. Eshagahi Gordji, M., Habibi, H.: Fixed point theory in generalized orthogonal metric space. J. Linear Topol. Algebra 6 251-260 (2017)

14. Ali, A., Shah, K., Jarad, F., Gupta, V., Abdeljawad, T.: Existence and stability analysis to a coupled system of implicit type implusive boundary value problems of fractional-order differential equations. Adv. Differ. Equ. 2019, Article ID 101 (2019)

15. Shoaib, M., Abdeljawad, T., Sarwar, M., Jarad, F.: Fixed point theorems for multi-valued contractions in $b$-metric spaces with applications to fractional differential and integral equations. IEEE Access 7, Article ID 2938635 (2019)

16. Panda, S.K., Abdeljawad, T., Ravichandran, C.: A complex valued approach to the solutions of Riemann-Liouville integral, Atangana-Baleanu integral operator and nonlinear telegraph via fixed point method. Chaos Solitons Fractals 130, Article ID 109439 (2020)

17. Panda, S.K., Abdeljawad, T., Ravichandran, C.: Novel fixed point approach to Atangana-Baleanu fractional and $L_{p}$-Fredholm integral equations. Alex. Eng. J. 59, 1959-1970 (2020)

18. Panda, S.K., Abdeljawad, T., Swamy, K.K.: New numerical scheme for solving integral equations via fixed point method using distinct $(\omega, F)$-contractions. Alex. Eng. J. 59, 2015-2026 (2020)

19. Mlaiki, N., Zarrad, A., Souayah, N., Mukheimer, A., Abdeljawed, T.: Fixed point theorem in $M_{b}$-metric spaces. J. Math. Anal. 7(5), 1-9 (2016) 